\title{
BMJ Open Vitamin K-dependent anticoagulant use and level of anticoagulation control in sub-Saharan Africa: protocol for a retrospective cohort study
}

\author{
Julius Chacha Mwita (D) , ${ }^{1,2}$ Albertino Damasceno, ${ }^{3}$ Pilly Chillo (D) ${ }^{4}$ \\ Okechukwu S Ogah, ${ }^{5}$ Karen Cohen, ${ }^{6}$ Anthony Oyekunle, ${ }^{7}$ Endale Tefera, ${ }^{8}$ \\ Joel Msafiri Francis (1) , ${ }^{9}$ On behalf of AAREN Investigators
}

To cite: Mwita JC, Damasceno A, Chillo P, et al. Vitamin K-dependent anticoagulant use and level of anticoagulation control in sub-Saharan Africa: protocol for a retrospective cohort study. BMJ Open 2022;12:e057166. doi:10.1136/ bmjopen-2021-057166

- Prepublication history for this paper is available online. To view these files, please visit the journal online (http://dx.doi. org/10.1136/bmjopen-2021057166).

Received 06 September 2021 Accepted 05 January 2022
Check for updates

(C) Author(s) (or their employer(s)) 2022. Re-use permitted under CC BY-NC. No commercial re-use. See rights and permissions. Published by BMJ.

For numbered affiliations see end of article.

Correspondence to Dr Julius Chacha Mwita; mwitajc@ub.ac.bw

\section{ABSTRACT}

Background Given that vitamin K-dependent anticoagulants (VKAs) will continue to be the primary anticoagulant in Africa for a long time, understanding the quality of anticoagulation services in the continent is vital for optimising the intended benefits. Notably, a few small studies have assessed the quality of anticoagulation in sub-Saharan Africa (SSA) countries. This study will describe the current VKA use and anticoagulation control among patients in selected SSA countries.

Methods and analysis We plan to review the 2019 anticoagulation data of a cohort of 800 random patients from 19 selected clinics in Botswana, the Democratic Republic of Congo, Ethiopia, Gambia, Ghana, Mozambique, Nigeria, Tanzania and South Africa. We expect at least one participating site to enrol 100 participants in each country. Eligible participants will be those on VKAs for at least 3 months and with at least four international normalised ratio (INR) results. We will document the indications, type and duration of VKA use, sociodemographic factors, coexisting medical conditions, concurrent use of drugs that interact with warfarin and alcohol and tobacco products. The level of anticoagulation control will be determined by calculating the time-in-therapeutic range (TTR) using the Rosendaal and the Percent of INR in TTR methods. A TTR of less than $65 \%$ will define a suboptimal anticoagulation control.

Ethics and dissemination This study was approved by the Ministry of Health and Wellness Ethics Committee (HPDME13/8/1) in Botswana and local research ethics committees or institutional review boards of all participating sites. As the study collects data from existing records, sites applied for waivers of consent. We will disseminate research findings through peer-reviewed scientific publications.

\section{INTRODUCTION}

Vitamin K-dependent anticoagulants (VKAs) continue to be the principal anticoagulants for treating and preventing thromboembolism in Africa. They produce their anticoagulant effects by interfering with the cyclic interconversion of vitamin $\mathrm{K}$ and its 2,3
Strengths and limitations of this study

- This continental study will provide insights into the types of vitamin K-dependent anticoagulants (VKAs) and anticoagulation control levels in sub-Saharan Africa (SSA).

- By providing comparable data from countries in SSA, the study will inform the delivery of care for patients on VKA.

- The study is limited by the non-random sampling of the included countries and participating sites.

- The retrospective design will potentially exclude patients with missing data.

epoxide, thus inhibiting $\gamma$-carboxylation of glutamate residues at the amino-terminals of coagulation factors II (prothrombin), VII, IX and $\mathrm{X}$, as well as of the anticoagulant proteins $\mathrm{C}, \mathrm{S}$ and $\mathrm{Z} .{ }^{12}$ As a result, VKA induces hepatic production of partially decarboxylated coagulation factors with reduced coagulant activity. ${ }^{2}$ While warfarin is the most widely used VKA, other anticoagulants in this group include acenocoumarol, phenprocoumon and phenindione. ${ }^{3}$

Because of their narrow therapeutic window, considerable variability in doseresponse among patients, and multiple interactions with other drugs and diet, the use of VKAs is generally tricky. ${ }^{1}$ It is often challenging to balance the risk of bleeding and, at the same time, prevent clotting. ${ }^{4}$ As therapeutic or prophylactic benefits of VKAs are dependent on their maintenance within therapeutic ranges (TTR), coagulation monitoring and frequent VKA dose adjustments are necessary. ${ }^{5}$

The VKA coagulation monitoring is achieved through a systematic international normalised ratio (INR) measurement. ${ }^{5}$ The INR is used to standardise prothrombin time 
and determine the degree to which VKA has successfully suppressed the patient's coagulation system. ${ }^{3}$ The target INR recommended for most warfarin indications is 2.0-3.0 (venous thromboembolism and cardiac arrhythmia) and 2.5-3.5 for mechanical prosthetic valves. ${ }^{2}{ }^{3}$ Sustaining a patient in TTR reduces the risk of thromboembolism while minimising the risk of bleeding attributable to excessive anticoagulation. On the other hand, the more time spent with INR below or above the TTR, the higher the risk of thromboembolic and bleeding complications, respectively. ${ }^{6}$ Consequently, the percentage of time spent in the TTR is a way of summarising anticoagulation control over time. Guidelines recommend a TTR above $65 \%$ as an optimal anticoagulation level to prevent thromboembolism without excessive risk of bleeding. ${ }^{6} \mathrm{~A}$ TTR above $65 \%$ is beneficial against stroke and vascular events among patients on warfarin, whereas a TTR of less than $40 \%$ is not associated with any significant mortality benefit. ${ }^{67}$

Due to multiple factors, a substantial proportion of warfarin-treated patients is sub-optimally anticoagulated..$^{8-11}$ These factors include the frequency of INR monitoring, health systems, and common polymorphisms influencing the pharmacokinetics or pharmacodynamics of VKAs. ${ }^{2811-13}$ Owing to the underfunding of the health systems in Africa, patients travel a long distance to access the few unreliably running centralised VKA services. ${ }^{14}$ Poor accessibility and out-of-pocket expenses for VKA services significantly impact anticoagulation control in these settings. ${ }^{101516}$ As a result, the proportion of patients on VKAs with optimal anticoagulation control in subSaharan Africa (SSA) is $0 \%-41 \% .^{1015-17}$

Given the high burden of rheumatic heart disease, mechanical heart valves and valvular atrial fibrillation are the main indications for long-term anticoagulation in SSA. Although non-vitamin $\mathrm{K}$ oral anticoagulants are apparent alternatives to VKA therapy for many indications (except mechanical valves and valvular atrial fibrillation), their uptake in African settings is limited by their high cost. Consequently, VKAs are likely to remain the primary anticoagulant in SSA for decades. As a suboptimal anticoagulation control is associated with an increased risk of thromboembolism, major bleeding and all-cause mortality, efforts to achieve optimal VKA anticoagulation are crucial in SSA. ${ }^{10}$ There is an urgent need to understand the extent of VKA regimen use and performance (anticoagulation control) in SSA. We aim to quantify the proportion of the patients taking VKA whose level of anticoagulation is below optimal thresholds, serving as a guide for targeting VKA anticoagulation control efforts.

\section{Aims and objectives}

Our overall objective is to describe the current VKA use and the quality of anticoagulation control among patients in SSA countries.

Our study has three specific objectives:

1. To describe types of VKA used by patients on long-term anticoagulation in selected SSA countries.
2. To assess and compare anticoagulation control for patients on VKAs in selected SSA countries.

3. To identify factors associated with optimal anticoagulation among patients of VKA in selected SSA countries.

\section{METHODS AND ANALYSIS}

Study design and patients

This will be a retrospective cohort study that reviews patient records at identified facilities in selected SSA countries. We will collect data from records of adult patients aged above 18 years who were on VKAs and attended the outpatient clinics from January to December 2019. The study will include all sampled patients who have attended the clinics during the specified period. Eligible participants will be those on VKAs for at least 3 months and with at least four INR results.

\section{Study setting}

This study will be conducted at clinics in the following countries: Botswana, the Democratic Republic of Congo, Ethiopia, Gambia, Ghana, Mozambique, Nigeria, Tanzania and South Africa (table 1).

\section{Sample size and sampling}

With a sample size of 100 participants from at least one participating site in each country, a minimum sample size of 800 , we will estimate a TTR of $41 \%$ with a margin of error of $3.5 \%$ on a two-sided alpha level of $0.05 .{ }^{16}$ We will apply a simple random sampling approach using random numbers generated by Excel/Stata to identify and select case records in the clinics with high volumes of patients.

\section{Data collection procedures}

Site PIs from each site will collect study data from patients charts and electronic medical records.

Using a standardised data extraction tool, they will collect information related to indications, type, and duration of VKA use. Sociodemographic factors, and if available, information on the coexisting medical conditions, concurrent use of drugs that interact with warfarin, and alcohol and tobacco products will be documented. Other data include INR values for the 12 months (January to December 2019), dates of INR testing and corresponding warfarin dosages. The level of anticoagulation will be determined by the time-in-TTR using the Rosendaal and the per cent of INR in TTR methods. ${ }^{5} 18$ The Rosendaal method assumes a linear change in INR values between consecutive testing time points. It calculates the proportion of time spent by a patient with INR in the TTR within the TTR. ${ }^{18}$ The method includes only patients who have at least two valid intervals for calculating per cent time in TTR. ${ }^{18} \mathrm{~A}$ valid interval consists of two INR values separated by 56 days ( 8 weeks) or less, without an intervening hospitalisation. For centres where INR testing is done at intervals of more than 56 days, the anticoagulation control level will be calculated using the per cent of INR in the TTR method. The method calculates the percentage of INR in the TTR for all patients divided by 
Table 1 Study sites for vitamin K-dependent anticoagulant (VKA) use and level of anticoagulation control in sub-Saharan Africa

\begin{tabular}{lll}
\hline Country & Site(s) & Description \\
\hline Botswana & Princess Marina Hospital $(\mathrm{PMH})$ & $\begin{array}{l}\text { PMH is a teaching hospital for the University of Botswana. The warfarin clinic runs } \\
\text { once a week, serving patients with different conditions }\end{array}$ \\
DRC & $\begin{array}{l}\text { Les Cliniques Universitaires de } \\
\text { Kinshasa }\end{array}$ & $\begin{array}{l}\text { This is a teaching hospital of the School of Medicine at the University of Kinshasa. } \\
\text { Patients on anticoagulants attend the Cardiology Unit of the Internal Medicine } \\
\text { Department. }\end{array}$
\end{tabular}

$\begin{array}{ll}\text { Ethiopia } & \text { Tikur Anbessa Specialised Hospital, } \\ \text { College of Health Sciences, Addis } \\ \text { Ababa University }\end{array}$

Ghana Greater Accra Regional Hospital and the Korlebu Teaching Hospital

$\begin{array}{ll}\text { Mozambique } & \text { Maputo Central Hospital } \\ \text { Nigeria } & \text { The University College Hospital, Ibadan } \\ & \text { The Federal Teaching Hospital Gombe } \\ & \text { Aminu Kano Teaching Hospital } \\ & \text { The Cardia Heart Clinic, Kano } \\ & \text { University of Nigeria Teaching Hospital } \\ & \text { Enugu } \\ & \text { The Federal Medical Centre Umuahia, } \\ & \text { Abia State, Nigeria. } \\ & \text { University of Uyo Teaching Hospital } \\ & \text { (UUTH) } \\ & \text { University of Port Harcourt Teaching } \\ & \text { Hospital } \\ & \text { Goodheart Medical Consultants }\end{array}$

The study will be conducted in adult and paediatric cardiac clinics.

The Greater Accra Hospital is a tertiary hospital in Accra, the capital city of Ghana Physician specialist clinics are run from Mondays to Fridays with an average of 40 patients daily. The Korlebu Teaching Hospital is a tertiary referral centre in Accra and runs a cardiology clinic once a week with an average attendance of 50 patients.

It is the university hospital of the faculty ofMedicine of Eduardo Mondlane University The people who are anticoagulated are followed in the cardiology outpatient clinic.

This is a teaching hospital affiliated with the University of Ibadan. Patients on VKA attend the anticoagulation clinic of the Haematology Department.

A tertiary hospital in the north-eastern Nigeria Patients on VKA, often for Valvular AF, attend the once-weekly Cardiology clinic.

This is a tertiary-level m public hospital in Kano. Patients attend the cardiology clinic once weekly.

This is a privately-owned cardiology clinic in Kano. VKA services run daily.

The National Centre for Cardiothoracic Medicine and Surgery in Enugu has an anticoagulation clinic that runs twice weekly.

Patients on VKA attends the Cardiology outpatient clinic once weekly.

UUTH is a tertiary-level multispeciality public hospital in Uyo, Nigeria. The study will be conducted at the cardiology outpatient clinics that runs twice a week.

A teaching hospital with weekly VKA service in the medical outpatient clinic on Tuesdays.

This is a private facility in the heart of Port Harcourt that has VKA services every day of the week.

$\begin{array}{ll}\text { Tanzania } & \text { Bugando Medical Cantre (BMC) } \\ \text { Muhimbili National Hospital (MNH) } & \\ \text { South Africa } & \begin{array}{l}\text { Thelle Mogoerane (formerly Natalspruit) } \\ \text { Regional Hospital. }\end{array}\end{array}$

Tambo Memorial Regional HospitalBoksburg

Groote Schuur Hospital
$\mathrm{BMC}$ is a regional referral and university teaching hospital in Mwanza. Patients on VKA attend the haematology and cardiology outpatient clinics.

MNH is a national and teaching hospital for Muhimbili university of health and Haematology and Blood Transfusion and runs once per week.

The hospital is situated in Vosloorus in the South sub-district of Ekurhuleni Metropolitan Municipality in Gauteng, South Africa. The hospital offers several services, including family medicine and primary care.

The hospital is situated in Boksburg in the North sub-district of Ekurhuleni Metropolitan Municipality in Gauteng, South Africa, linked to the University of Witwatersrand medical school in Johannesburg.

A tertiary academic hospital in Cape Town, South Africa. The hospital has an anticoagulation clinic . allied sciences. The anticoagulation clinic is housed within the Department of

AF, Atrial fibrillation; BMC, Bugando Medical Cantre; DRC, Democratic Republic of Congo; MNH, Muhimbili National Hospital; PMH, Princess Marina Hospital; UUTH, University of Uyo Teaching Hospital ; VKA, Vitamin K-dependent anticoagulant.

the overall number of INRs during the period. ${ }^{5}$ Therapeutic INR will be defined as 2.0-3.0 for venous thromboembolism and cardiac arrhythmia and 2.5-3.5 for mechanical prosthetic valves. ${ }^{3}$ A TTR of less than $65 \%$ will be considered suboptimal anticoagulation control. While some sites have finished data collection, most will likely continue data extraction for the next 6 months (up to roughly June 2022).

\section{Statistical analysis}

Data will be entered in REDCap, hosted by the University of Botswana. Clean data will be analysed using Stata V.16 (Stata). Categorical variables (sociodemographic factors, anticoagulation type, VKA indication, comorbidities, other drug use, alcohol and tobacco products use) will be presented as frequencies and percentages, and continuous variables (duration on VKA, INR estimates) as mean 
(SD) or medians (first-third quartiles). Comparison of categorical variables and the level of anticoagulation control will be made using the $\chi^{2}$ or Fisher's exact tests, and independent Student's t-tests or Wilcoxon rank-sum test for continuous variables, as appropriate. A two-sided $\mathrm{p}<0.05$ will be considered statistically significant. A multivariate logistic regression will be performed to assess independent predictors for anticoagulation control. The multivariable model will include all factors with a $\mathrm{p}<0.2$ on bivariate analysis. A backward selection modelling method with probabilities set at 0.05 and 0.1 for inclusion and exclusion, respectively, will be used. Adjusted ORs, 95\% CIs, and $\mathrm{p}$ values will be documented.

\section{Patient and public involvement}

Participants were not invited to participate in the study design. However, research findings will be disseminated through presentations to users and advocacy groups in different countries.

\section{ETHICS AND DISSEMINATION}

Patient consent for publication

Not required.

\section{Ethical considerations}

The Ministry of Health and Wellness of Botswana granted ethical approval for the University of Botswana/Princess Marina as a coordinating centre (HPDME13/8/1). Site principal investigators (PIs) were responsible for obtaining necessary ethical approvals from local research ethics committees or institutional review boards of all participating sites-Muhimbili National hospital, Tanzania (MNH/TRCU/Perm/2021/012); the University of Cape Town, South Africa (HREC:047/2021); the University of the Witwatersrand Johannesburg, South Africa (R14/49); Catholic University of Allied Sciences Bugando, Tanzania (CREC/445/2020/); Federal Medical Centre, Umuahia, Nigeria (FMC/QEH/G/596/ VOL.10/485); University of Ibadan College of Medicine (UI/EC/20/0349; Korle Bu Teaching Hospital Institutional Review Board (KBCH/MD/G3/20); University of Port Harcourt Teaching Hospital (UPTH/ADM/90/5. II/VOL XI/959; University of Nigeria Teaching Hospital, Nigeria (UNTH/CSA/329/VOL.5/09; Federal Teaching Hospital Gombe, Nigeria (NHREC/25/10/2013); Amino Kano Teaching Hospital Nigeria (AKTH/MAC/ SUB/12 A/P-3/VI/2989); Les Cliniques Universitaires de Kinshasa (213/CNES/BN/PMMF/2020); University of Uyo Teaching Hospital; College of Health Sciences, Addis Ababa University, Ethiopia (115/20/IM) and Faculty of Medicine, University Eduardo Mondlane, Mozambique, the Gambia Government/MRCG Joint Ethics Committee (22803). As the study will collect data from existing records and not engage living human subjects, sites applied for waivers of consent.

\section{Data management}

The overall PI will coordinate the data management process. Site PIs will collect information in an electronic case report form (CRF) or a paper-based CRF during internet connection problems. The site PIs will be responsible for routine supervision of the data entry into a password-protected Research Electronic Data Capture (REDCap) database, with the University of Botswana assistance as necessary. Extracted data will be anonymised by removing any identifying details. Except for the overall PI, who will have access to all the data, site researchers will only have access to their site-specific data. Although the collaborators will jointly own the data generated, the PI will coordinate data sharing and archiving.

\section{Dissemination policy and plans}

The findings will be disseminated via publications in peer-reviewed journals, conferences presentations and other forms of public engagement. Summary reports will be submitted to the participating institutions. Copies of all published materials and reports will be shared with the research ethics committees and collaborators. The results will also be shared with policy-makers and stakeholders, highlighting any identified gaps in VKA use in SSA. We will also make our findings available in accessible formats to patient groups and relevant charities. Site PIs from each site that contribute to patients will coauthor on any resulting publications.

\section{Author affiliations}

${ }^{1}$ Internal Medicine, University of Botswana, Gaborone, South-East District, Botswana ${ }^{2}$ Internal Medicine, Princess Marina Hospital, Gaborone, Botswana

${ }^{3}$ Internal Medicine, University of Eduardo Mondlane Faculty of Medicine, Maputo, Mozambique

${ }^{4}$ Internal Medicine, Muhimbili University of Health and Allied Sciences, Dar es Salaam, Tanzania, United Republic of Tanzania

${ }^{5}$ Internal Medicine, University of Ibadan College of Medicine, Ibadan, Oyo, Nigeria ${ }^{6}$ Clinical Pharmacology, University of Cape Town Faculty of Health Sciences, Anzio Road, Observatory 7925 Cape Town, South Africa

${ }^{7}$ Internal Medicine, Bugando Medical Centre, Mwanza, Mwanza, Tanzania, United Republic of Tanzania

${ }^{8}$ Paediatrics and Adolescent Health, University of Botswana, Gaborone, South-East District, Botswana

${ }^{9}$ Family Medicine and Primary Care, University of the Witwatersrand School of Social Sciences, Johannesburg, South Africa

Twitter Joel Msafiri Francis @joframsa

Acknowledgements We thank the African Anticoagulation Research and Education Network (AAREN) for their valuable inputs in the planning and providing the multinational perspective on the current study. Besides the authors, other members of the AAREN group are Karen Forrest, Francis Agyekum, Wigilya Mikomangwa, Aba Ankomaba Folson, Ayoola, Yekeen Ayodele, Kamilu Karaye Raphael Anakwue, Shogade Tolulope Taiwo, Leodegard Mujwahuzi, Eze Nwafor, Chiedozie Maduka, Jean-Pierre Fina, Tsie Kabo, Dejuma Yadete, Hannes Mouton, Blessing Enyichukwu, Kagiso Ndlovu and John Musonda.

Collaborators Anticoagulation Research and Education Network (AAREN) 1. Karen Forrest 2. Francis Agyekum 3. Aba Ankomaba Folson 4. Ayoola, Yekeen Ayodele 5. Kamilu Karaye 6. Raphael Anakwue 7. Shogade Tolulope Taiwo 8. Leodegard Mujwahuzi 8. Eze Nwafor 9. Chiedozie Maduka 10. Jean-Pierre Fina 11. Tsie Kabo 12. Dejuma Yadete 13. Hannes Mouton 14. Blessing Enyichukwu 15. Wigilya Mikomangwa16. Kagiso Ndlovu 17. John Musonda

Contributors JCM is the principal investigator who conceived the study and drafted the protocol. AD, KC, PC, OSO and JMF are co-principal investigators of the study and have contributed to manuscript drafts and approved the final draft. $\mathrm{A} 0$ and $\mathrm{ET}$ developed the data collection tool and contributed to the review and approval of the final draft. 
Funding The authors have not declared a specific grant for this research from any funding agency in the public, commercial or not-for-profit sectors.

Disclaimer The views expressed in this publication are those of the author(s) and not necessarily those of AAREN.

Competing interests None declared.

Patient and public involvement Patients and/or the public were not involved in the design, or conduct, or reporting, or dissemination plans of this research.

Patient consent for publication Not applicable.

Provenance and peer review Not commissioned; externally peer reviewed.

Open access This is an open access article distributed in accordance with the Creative Commons Attribution Non Commercial (CC BY-NC 4.0) license, which permits others to distribute, remix, adapt, build upon this work non-commercially, and license their derivative works on different terms, provided the original work is properly cited, appropriate credit is given, any changes made indicated, and the use is non-commercial. See: http://creativecommons.org/licenses/by-nc/4.0/.

\section{ORCID iDs}

Julius Chacha Mwita http://orcid.org/0000-0002-5947-3684

Pilly Chillo http://orcid.org/0000-0001-7073-1122

Joel Msafiri Francis http://orcid.org/0000-0003-1902-2683

\section{REFERENCES}

1 De Caterina R, Husted S, Wallentin L, et al. Vitamin K antagonists in heart disease: current status and perspectives (Section III). Position paper of the ESC Working Group on Thrombosis--Task Force on Anticoagulants in Heart Disease. Thromb Haemost 2013;110:1087-107.

2 Hirsh J, Fuster V, Ansell J, et al. American heart Association/ American College of cardiology Foundation guide to warfarin therapy. J Am Coll Cardiol 2003;41:1633-52.

3 Hirsh J, Poller L, Deykin D, et al. Optimal therapeutic range for oral anticoagulants. Chest 1989;95:5S-11.

4 Ansell J, Hirsh J, Poller L, et al. The pharmacology and management of the vitamin K antagonists: the seventh ACCP conference on antithrombotic and thrombolytic therapy. Chest 2004;126:204s-33.

5 Kaatz S. Determinants and measures of quality in oral anticoagulation therapy. J Thromb Thrombolysis 2008;25:61-6.
6 Connolly SJ, Pogue J, Eikelboom J, et al. Benefit of oral anticoagulant over antiplatelet therapy in atrial fibrillation depends on the quality of international normalized ratio control achieved by centers and countries as measured by time in therapeutic range. Circulation 2008;118:2029-37.

7 Morgan CL, McEwan P, Tukiendorf A, et al. Warfarin treatment in patients with atrial fibrillation: observing outcomes associated with varying levels of Inr control. Thromb Res 2009;124:37-41.

8 van Walraven $\mathrm{C}$, Jennings $\mathrm{A}$, Oake $\mathrm{N}$, et al. Effect of study setting on anticoagulation control: a systematic review and metaregression. Chest 2006;129:1155-66.

9 Sarawate C, Sikirica MV, Willey VJ, et al. Monitoring anticoagulation in atrial fibrillation. J Thromb Thrombolysis 2006;21:191-8.

10 Haas S, Ten Cate H, Accetta G, et al. Quality of vitamin K antagonist control and 1-year outcomes in patients with atrial fibrillation: a global perspective from the GARFIELD-AF registry. PLoS One 2016;11:e0164076.

11 Mearns ES, White CM, Kohn CG, et al. Quality of vitamin K antagonist control and outcomes in atrial fibrillation patients: a metaanalysis and meta-regression. Thromb J 2014;12:14.

12 Singer DE, Hellkamp AS, Piccini JP, et al. Impact of global geographic region on time in therapeutic range on warfarin anticoagulant therapy: data from the rocket AF clinical trial. J Am Heart Assoc 2013;2:e000067-e67.

13 Aithal GP, Day CP, Kesteven PJ, et al. Association of polymorphisms in the cytochrome P450 CYP2C9 with warfarin dose requirement and risk of bleeding complications. Lancet 1999;353:717-9.

14 Barth DD, Zühlke LJ, Joachim A, et al. Effect of distance to health facility on the maintenance of Inr therapeutic ranges in rheumatic heart disease patients from Cape town: no evidence for an association. BMC Health Serv Res 2015;15:219-19.

15 Mwita JC, Francis JM, Oyekunle AA, et al. Quality of anticoagulation with warfarin at a tertiary hospital in Botswana. Clin Appl Thromb Hemost 2018;24:596-601.

16 Semakula JR, Mouton JP, Jorgensen A, et al. A cross-sectional evaluation of five warfarin anticoagulation services in Uganda and South Africa. PLoS One 2020;15:e0227458.

17 Ebrahim I, Bryer A, Cohen K, et al. Poor anticoagulation control in patients taking warfarin at a tertiary and district-level prothrombin clinic in Cape town, South Africa. S Afr Med J 2018;108:490-4.

18 Rosendaal FR, Cannegieter SC, van der Meer FJ, et al. A method to determine the optimal intensity of oral anticoagulant therapy. Thromb Haemost 1993;69:236-9. 